\title{
Desigualdades Estructurales, Salud de Jóvenes LGBT y Lagunas de Conocimiento: ¿Qué Sabemos y qué Preguntamos?
}

\author{
Mario Pecheny ${ }^{1}$ \\ Facultad de Ciencias Sociales de la Universidad de Buenos Aires, Buenos Aires, Argentina \\ Instituto Gino Germani del Consejo Nacional de Investigaciones Científicas y Técnicas, \\ Buenos Aires, Argentina
}

\begin{abstract}
Resumen
Hay estudios que examinan los vínculos entre desigualdades estructurales y vulnerabilidad en salud, y algunos de ellos lo hacen en relación con la vulnerabilidad de la población LGBT. Pero pocos examinan la especificidad de LGBT en tanto generación joven. Las y los jóvenes LGBT son particularmente vulnerables a enfermedades, como el VIH/sida y otras infecciones de transmisión sexual o a padecimientos de salud mental, y a exponerse a violencias y circunstancias de vida complicadas. Este trabajo de tono especulativo interroga cómo entender el papel productor de riesgos y daños que tienen las desigualdades, y cómo son los procesos concretos en que se dan estos fenómenos. ¿Qué dice la literatura internacional sobre el impacto de los contextos de alta desigualdad socio-económica en las vidas de jóvenes homosexuales, particularmente en su salud? ¿Hay tecnologías de promoción de la salud en relación con tales contextos? Una primera revisión de la literatura identifica una matriz individualista en las concepciones de génesis de los problemas, de la investigación y de las respuestas. En cambio, se propone eludir las epistemologías positivistas que brindan pinturas parciales de los fenómenos que pretenden describir y comprender; $y$ en cuanto a técnicas de intervención, pensar técnicas eficaces que superen una ingeniería social fragmentaria.
\end{abstract}

Palabras clave: Salud, desigualdad, juventud, LGBT, vulnerabilidad.

\section{Structural Inequalities, LGBT Youth Health and Knowledge Gaps: What do we Know and what do we Ask?}

\begin{abstract}
Numerous studies have examined the links between structural inequalities and health vulnerability. Some of them have done so in relation to LGBT people. However, few studies have examined the specificity of LGBT youth. LGBT youth are particularly vulnerable to some illnesses, such as HIV/AIDS and other STIs, mental health problems, violence. This speculative paper rise questions about how to understand inequalities as producing risk and harms, the concrete processes the go from structures to outcomes. What does international literature say about the impact of high socio-economic inequalities on the lives of homosexual youths, especially on their health? Are there health promotion technologies adequate to those contexts? A quick review of literature identifies an individualistic matrix presupposed to explain the genesis, research and answers when dealing with these problems. The text invites to overcome positivistic epistemologies that provide only partial pictures and support techniques that are
\end{abstract}

Dirección para correspondencia: Instituto Gino Germani, Facultad de Ciencias Sociales, Universidad de Buenos Aires, J. E. Uriburu 950 6to. Piso, 1114, Buenos Aires, Argentina. E-mail: mpecheny@retina.ar. 
reduced to "fragmentary engineering". More comprehensive and situated epistemologies might support more efficient techniques to address risks and harms produced by structural inequalities.

Keywords: Health, inequalities, youths, LGBT, vulnerability.

\section{Desigualdades Estruturais, Saúde de Jovens LGBT e Lacunas de Conhecimento: Que Sabemos e que Perguntamos?}

\section{Resumo}

Há estudos que examinam os vínculos entre as desigualdades estruturais e a vulnerabilidade em saúde, e alguns deles o fazem em relação à vulnerabilidade da população LGBT. Mas, poucos examinam a especificidade de LGBT enquanto geração jovem. As e os jovens LGBT são particularmente vulneráveis a doenças, como HIV/AIDS e outras infecções sexualmente transmissíveis, ou a padecimentos de saúde mental, e a se expor a violências e circunstâncias de vida complicadas. Este trabalho especulativo interroga sobre como entender o papel produtor de riscos e danos que têm as desigualdades; e como são os processos concretos em que se dão esses fenômenos. O que diz a literatura internacional sobre o impacto dos contextos de alta desigualdade socioeconômica nas vidas de jovens homossexuais, particularmente em sua saúde. Existem tecnologias de promoção da saúde relacionadas a esses contextos? Uma primeira revisão da literatura identifica uma matriz individualista nas concepções da gênese dos problemas, da pesquisa e das respostas. Em outra direção, este texto se propõe a escapar às epistemologias positivistas que oferecem pinturas parciais dos fenômenos que pretendem descrever e compreender; e em relação a técnicas de intervenção, pensar técnicas eficazes que superem uma engenharia social fragmentaria.

Palavras-chave: Saúde, desigualdade, juventude, LGBT, vulnerabilidade.

¿Qué dice la literatura internacional sobre el impacto de los contextos de alta desigualdad socio-económica en las vidas de jóvenes homosexuales, particularmente en su salud? ¿Hay tecnologías de promoción de la salud en relación con tales contextos? Estas son las preguntas disparadoras para este artículo, considerado como un primer acercamiento (del autor) al tema específico. En realidad es un tema específico, pero no porque sea preciso y focalizado, sino porque concentra, cual haz de luz, una intersección de variables sustantivas y miradas analíticas.

La población sobre la que tratan estas páginas es la de jóvenes cuyas prácticas, expresiones, vínculos e identidades se apartan de la normatividad heterosexual; por comodidad, para referirnos a este segmento hablaremos de jóvenes LGBT, es decir lesbianas, gays, bisexuales $\mathrm{y}$ trans, aunque en otros momentos del texto nos interesen más las expresiones que las identidades de género y sexualidad.

Este texto mantiene el estilo interrogativo del título, y su pretensión es más bien conjetural y especulativa, en la idea de que no es volumen de información lo que falta sino más bien avanzar en preguntas y abordajes que consideramos más pertinentes.

Pensar el campo problemático de la salud juvenil en la intersección de las desigualdades económicas, sociales, de género, etarias y sexuales (y raciales, regionales, etc.), pensadas como desigualdades estructurales, y en relación con quienes se apartan de las normas de género y sexuales heterosexuales, trae una serie de desafíos.

Un desafío es escaparle a afirmaciones que pudieron haber sido útiles en algún momento, y que siguen siéndolo en ciertos entornos, pero que son demasiado generales o abstractas como para a partir de ahí diseñar y poner en marcha intervenciones. Afirmar, por ejemplo, que no existen factores sociales que sean externos a cualquier fenómeno de salud que elijamos. Sabemos que los factores sociales no son "factores" ni son externos, sino que determinan, o producen, al fenómeno en cuestión (Auerbach, Parkhurst, \& Cáceres, 2011; Padilla, Vázquez del Aguila, \& Parker, 2007). Entonces, ¿cómo estudiar los vínculos entre lo social y la salud más concretamen- 
te? Si todo fenómeno responde a fuerzas constitutivas de diversos órdenes, es necesario estudiar empíricamente, para cada caso, las modalidades en que estas fuerzas se refuerzan, se yuxtaponen, se neutralizan o quizá se contradicen (Stall, Friedman, \& Catania, 2007; Wong, Weiss, Ayala, \& Kipke, 2010).

En una primera mirada, a diferencia de otras categorías sociales, no parece haberse hecho este esfuerzo por determinar cuánto y cómo las desigualdades socio-económicas determinan la salud en particular de las y los jóvenes LGBT, a través de qué procesos y mecanismos, con qué resistencias y complicidades. Los estudios sobre LGBT encontrados generalmente se focalizan en conductas individuales de riesgo para la salud que pueden ser alentadas o mitigadas por instituciones, relaciones, etc., pero que se consideran de hecho en una situación de exterioridad con esos riesgos y daños para la salud.

Aunque no necesariamente sea sentido común, tampoco es muy novedoso afirmar que las desigualdades socio-económicas, de género, sexuales y otras, sobre todo cuando son altas y persistentes como en América Latina y otras regiones del mundo, impactan significativamente en la vida de las personas más vulnerables, y que este impacto no es exterior sino que justamente produce tales vulnerabilidades: y que por eso consideramos esas vulnerabilidades estructurales, y no resultado de actitudes personales, familiares o comunitarias, de conocimientos y prácticas, de acceso circunstancial a determinados recursos e instituciones. Esto también ya es, de alguna manera, sabido.

Lo novedoso puede ser encarar estas cuestiones buscando patrones específicos de relaciones y pensando en intervenir. Dentro de este marco de preocupaciones, las preguntas disparadoras desde la organización del seminario aportaron especificidad y originalidad en tres aspectos poco o nada explorados: (a) la población sobre la cual pensar y estudiar estas cosas: es decir, niños/as y jóvenes LGBT (Remafedi, 2007); (b) el modo de pensar y estudiar: como un proceso complejo e integrado que es sólo desagregable para un momento "dialéctico" del análisis, pero que se considera sustancialmente agregado y analíticamente completo solo si se compone la totalidad (Habermas, 1988); (c) la voluntad intelectual y política de ir dos pasos más allá de la descripción y explicación de los fenómenos: un paso es pensar y diseñar tecnologías de intervención que den cuenta de esta población y de estos procesos; un segundo paso es evaluar las condiciones de aplicación y los resultados de esas tecnologías (Paiva, Ayres, \& Buchalla, 2012).

El concepto de vulnerabilidad invita a esforzarnos por pensar los fenómenos como formando parte de, y siendo producidos por, la totalidad social (Ayres, Paiva, \& França, 2012), más allá de la concepción epidemiológica de riesgo que se traduce en enfocar individuos, grupos y comportamientos identificables, definidos en última instancia a partir de asociaciones probabilísticas, y por ende calculables, entre determinadas condiciones mensurables, uno o más agentes transmisores o causantes, y algún daño o perjuicio también discernible y desagregable. Como puso de relieve la experiencia del VIH/sida en los ochenta y noventa, la retraducción social del vocablo riesgo (en lenguaje experto, una medida de probabilidad) en términos de identificaciones sociales ("grupos de riesgo") e incluso de auto-identidades sociales, trajo más efectos de señalamiento de ciertas categorías, el estigma y la discriminación (Parker \& Aggleton, 2003), que de ayudar a la cuantificación de las infecciones, la morbilidad y la mortalidad. Si la noción de riesgo es analítica, en el sentido de desagregar agentes, fenómenos y efectos, la noción de vulnerabilidad pretende ser sintética, en el sentido de agregar los elementos aislados en totalidades relacionales complejas, y apuntando más a cierta inteligibilidad que a la calculabilidad (Ayres, França, Junqueira Calazans, \& Saletti, 2008).

Por eso "vulnerabilidad" no es un vocablo políticamente correcto que viene a reemplazar al "riesgo". El concepto de vulnerabilidad designa un conjunto de aspectos individuales y colectivos relacionados a la mayor susceptibilidad de individuos y comunidades a padecimientos o perjuicios $\mathrm{y}$, de modo inseparable, menor disponibilidad de recursos para su protección. Los análisis de la dimensión individual de la vulnerabilidad parten del individuo pero siempre en relación. La dimensión social apunta a los aspectos relacionales económicos, de género, raciales, 
generacionales, laborales, etc. que conforman vulnerabilidades individuales. La dimensión programática refiere a si, y en qué medida, los servicios de salud y el Estado en sentido más amplio enfrentan y/o reproducen las condiciones sistemáticas o puntuales de vulnerabilidad, en cada contexto local. La dimensiones son analíticas, no son separables (Ayres et al., 2012).

Esta perspectiva sobre vulnerabilidad es coherente con la perspectiva según la cual la desigualdad social no resulta de la agregación de desigualdades discernibles más allá del análisis, sino de dimensiones integradas que no sin tensiones se retroalimentan y reproducen: acceso y situación educativa, acceso y situación en relación con los mercados de trabajo, exclusión e inclusión en los programas sociales del Estado y otros, segregación espacial, estigma y discriminación integrados sinérgicamente (en materia de sexualidad, generación, estilos de vida, consumo, lenguajes...). En este sentido, tampoco el género es una dimensión más de la experiencia o la identidad individual ni colectiva, un atributo discernible de individuos autónomos, sino que refiere a una dimensión particular de las relaciones sociales, la dimensión sexuada (Illouz, 2009; Petchesky, 2007). Dicho de otra manera, el género no refiere a las identidades y experiencias de las mujeres en tanto mujeres, o de los varones en tanto varones, sino a las relaciones intra- y entre-sujetos en relación con sus identidades, experiencias y relaciones sexuadas y sexuales, incluyendo aquellas identidades, experiencias y relaciones no específicamente o explícitamente consideradas sexuales (Pecheny \& de la Dehesa, 2012).

Introduzco un primer y rápido recorrido por trabajos que sintetizan literatura que directa o indirectamente cubre algunos de los aspectos mencionados, para mostrar no tanto las ausencias de lo que no se hizo o no se hace (o, muy probablemente, yo no haya logrado encontrar) sino para mostrar cierta producción de la ausencia: qué presupuestos teórico-metodológicos, qué modos de conceptualizar y de hacer investigación, qué prioridades suelen establecerse a la hora de investigar e intervenir, que describan y expliquen estas hipotéticas (y probablemente reales) lagunas.
Asimismo, y en el ánimo conjetural que adelanté, plantearé una serie de preguntas o pistas surgidas a partir de estas lecturas.

\section{La Salud de Jóvenes LGBT y la Vulnerabilidad Social: Ejes Puestos en el VIH/Sida y las Actitudes y Vulnerabilidades Individuales}

No hace falta justificar que las y los jóvenes LGBT son particularmente vulnerables a enfermedades, como el VIH/sida y otras infecciones de transmisión sexual o a padecimientos de salud mental (como los trastornos de la alimentación o las ideaciones y prácticas suicidas), y a exponerse a violencias y circunstancias de vida complicadas. Cuando escribo "particularmente" vulnerables no quiero decir "más" vulnerables, pero sí "específicamente" vulnerables.

Los jóvenes LGBT tienen padecimientos específicos, no relacionados con morfologías corporales y cerebrales diferentes a las de las/ los jóvenes heterosexuales, sino con los contextos estigmatizantes y/o violentos (Altman et al., 2012), casi universalmente hostiles, que enfrentan. Hostilidad que tampoco es atribuible a morfologías biológicas sino a prácticas sociales, muchas de ellas muy institucionalizadas en jerarquías de género y en una heteronormatividad sistemática.

La literatura científica, pareciera ser, empezó a ocuparse de la salud de jóvenes LGBT sólo a partir de la epidemia del VIH/sida, y casi toda la producción es en relación con ella. De ahí que los gays, los bisexuales, los "hombres que tienen sexo con hombres", y las trans, en este caso jóvenes, hayan recibido mucha más atención que las lesbianas y mujeres bisexuales (Facchini \& Barbosa, 2006). También de ahí que casi exclusivamente la atención se haya enfocado en las ITS, y luego - más recientemente - en indagar sobre cuestiones de salud mental, uso de drogas, violencia y otras cuestiones consideradas propias o típicas de estas fases de la vida, donde se intenta discriminar las especificidades de niñas/os, adolescentes y jóvenes LGBT en el contexto de la generalidad presupuesta como heterosexual.

Datos de un Informe que resume los $U N$ GASS Country Progress Reports, 2010 (Joint 
United Nations Program on HIV/AIDS [UNAIDS], 2011, p. 19), en algunos países seleccionados, indican que en muestras de "HSH", jóvenes hombres de 25 años o menos que tienen sexo con hombres, para el período 2008-2010, la prevalencia del VIH es mayor que para los jóvenes heterosexuales de esos mismos países (aunque presumiblemente menor que para los $\mathrm{HSH}$ de más edad). Estos datos dan la pauta de que para la generación de HSH nacida ya con la epidemia de VIH/sida, la prevención todavía es una materia muy pendiente (Beyrer et al., 2012).

Para ese informe de lo Programa Conjunto de las Naciones Unidas sobre el VIH/Sida (ONUSIDA), reproduciendo una afirmación que ya también es de sentido común, "el riesgo y la vulnerabilidad de la gente joven son muy específicos a los contextos" (UNAIDS, 2011, p. 20). Por ello sugiere, entre otras cosas, relevar información y medir prevalencia de jóvenes de estas poblaciones clave de $\mathrm{HSH}$, así como de usuarios de drogas inyectables, que hacen trabajo sexual, etc. Esos y otros datos sobre categorías especialmente afectadas por el VIH muestran consistentemente y desde hace años que tales categorías siguen siendo ampliamente afectadas por el VIH; y que la franja de edad joven es clave.

La franja joven es clave en dos sentidos: primero, porque el ingreso a las prácticas de riesgo (sexo sin protección, uso compartido de equipos y sustancias) se hace en edades jóvenes o muy jóvenes (por ejemplo, dice ese Informe, la mayoría de las trabajadoras sexuales han comenzado con esta actividad antes de los 20 años de edad); segundo, por el hecho comprobable de que es muy elevada la proporción de las franjas jóvenes al interior de cada una de estas sub-poblaciones (por ejemplo ONUSIDA, 2006 señala que 70\% de los usuarios de drogas inyectables a nivel mundial tienen menos de 25 años).

Arriesgando una interpretación temeraria, la lectura de datos sobre jóvenes LGBT (y no sólo jóvenes, y no sólo LGBT) a lo largo de tantos años de epidemia de VIH/sida nos permite conjeturar que, no obstante las características idiosincráticas, hay patrones bastante gruesos que las particularidades a veces oscurecen: las grandes líneas de procesos históricos, y los fuertes y sostenidos clivajes de desigualdad y vulnerabili- dad, determinan propensiones diferenciales a infectarse, enfermarse y morirse prematuramente.

Decenas de estudios con jóvenes LGBT (y no sólo LGBT) muestran esto. Sin embargo, los estudios no suelen ir más allá de reflejar las perspectivas de estos jóvenes (sus informaciones, sus temores y niveles de auto-estima, los recursos con que cuentan) o sus características socio-demográficas, y suelen evitar pensar los contextos institucionales (familiares, urbanos, educativos, ligados al ocio y laborales) que no simplemente favorecen, sino producen y reproducen esas vulnerabilidades y sus consecuencias en términos de morbilidad y mortalidad.

¿Cuántos más estudios específicos son necesarios para explicar que la ilegalidad o estigmatización de algunas prácticas, expresiones e identidades sexuales y de género producen daño? ¿Cuántos más estudios específicos son necesarios para explicar que las desigualdades económicas y la pobreza producen daño? ¿Cuántos más estudios para mostrar que ambos procesos se refuerzan mutuamente?

Aquí es necesario, más bien, estudiar los "cómo" de esos procesos, en vistas a pensar en los modos de intervenir para transformar esas condiciones que producen y reproducen vulnerabilidad. Hay un salto que desde las investigaciones nos es difícil recorrer: los factores macro-sociales (económicos, políticos, de jerarquías de género y sexuales) están en un extremo, las condiciones micro (información, acceso a preservativos, etc.) están en el otro; no sabemos bien, y no sabemos bien cómo intervenir, acerca de las relaciones macro-micro y las mediaciones existentes. Cuesta investigar e intervenir "hegelianamente" sobre la totalidad de relaciones que producen al todo (Habermas, 1988), es decir considerando a cada proceso y a cada interacción como formando parte de una totalidad que conflictivamente los supone y les da sentido y existencia.

¿Cómo intervenir desde la política y desde la política pública? En relación con la actividad política, la ciencia y la investigación no son las más indicadas para proveer modos de intervención, en el marco de la política pensada como práctica democrática y no como técnica o acción puramente instrumental. Pero, no obstante, en 
relación con las políticas públicas de promoción de la salud, basadas en evidencia y en compromiso con los derechos de los sujetos, sí la ciencia y la investigación están llamadas a pensar, proponer y testear tecnologías, en una lógica instrumental pero que debe integrarse en un marco práctico y de reconocimiento de los distintos, y eventualmente conflictivos, proyectos subjetivos de felicidad (Ayres, 2002).

\section{Reflexiones sobre Algunos Trabajos de Sistematización sobre Salud de Jóvenes LGBT}

No voy a presentar un estado del arte o de la cuestión, sería deshonesto de mi parte sostener algo así. Lo que voy a presentar son algunos puntos de dos trabajos de sistematización que muestran las lagunas que refiere el título disparador, lagunas que tienen que ver menos con la aparentemente poca cantidad de estudios o propuestas sobre juventud LGBT y salud, o con la cantidad de datos disponibles, sino con modos privilegiados de aproximarse al tema, que lo hacen desde una matriz individual(ista), y consideran los procesos sociales a lo sumo como contextuales y no productores de los fenómenos.

El primer trabajo está limitado geográficamente a Estados Unidos y teórico-metodológicamente al ámbito de la salud pública, pero es exhaustivo en el recorte explicitado, es abarcador en el conjunto de problemáticas que incluye, y permite reconocer ciertos presupuestos y puntos de partida que no se dan solamente en Estados Unidos o solamente en la salud pública. Se trata del artículo The Health and Health Care of Lesbian, Gay and Bisexual Adolescents (Coker, Austin, \& Schuster, 2010), publicado en la $A n$ nual Review of Public Health.

La revisión bibliográfica (trabajos todos sobre Estados Unidos) "ha demostrado conductas de riesgo para la salud sustantivamente más riesgosas y con peores resultados para la salud en jóvenes LGB comparado con jóvenes heterosexuales" (Coker et al., 2010, p. 258), e incluye por ejemplo uso de drogas, trastornos de la alimentación, tendencia a suicidio, conductas sexuales de riesgo, exposición a la violencia y situación de calle.
El enfoque de esta revisión bibliográfica apunta a cuantificar diferencialmente la probabilidad de que jóvenes LGB (particularmente adolescentes, de 11 a 21 años) tengan conductas o sufran enfermedades o daños, en relación con el conjunto de los jóvenes. Los estudios considerados son generalmente surveys realizados para medir conocimientos, actitudes y prácticas en jóvenes escolarizados, y a veces no-escolarizados.

El contexto es presentado como amigable u hostil, y puede jugar en varios sentidos, pero aparece predominantemente en los trabajos como externo a los fenómenos de salud/enfermedad. Cuando aparece como "productor" de los fenómenos, la investigación parece reducirse a indagar cómo los sujetos perciben la hostilidad o amigabilidad de las instituciones y sujetos con los que interactúan. Poco y nada hay sobre la génesis y reproducción, los mecanismos de funcionamiento, y las posibilidades (o no) de transformar esas instituciones y esas relaciones intersubjetivas.

Lo interesante de la revisión es que muestra con sobrada evidencia que la discriminación directamente ejercida y/o anticipada por los sujetos (Pecheny, 2002) tiene efectos en la salud física y mental, en los comportamientos y hasta somáticamente en los individuos LGB. La mayoría de las veces los estudios relevan experiencias concretas e identificables en las que los individuos se sintieron discriminados o fueron víctimas de actos discriminatorios. Sin embargo, los estudios ponen muy raramente de relieve los mecanismos violentos y potencialmente dañinos de la presunción de heterosexualidad. Esta presunción opera como fuerte factor de exclusión y señalamiento, y no se reduce a actitudes de individuos o a normas reglamentadas sino que como trasfondo de sentido común y de lenguaje común de interacciones sociales deja siempre fuera de juego a quienes no comparten ese presupuesto dado por universal. De modo que mi pregunta recurrente es cómo se relacionan las desigualdades sociales y la presunción de heterosexualidad en riesgos, daños y estrategias de resistencia diferenciales (Altman et al., 2012).

Otro aspecto interesante es resaltar el peso de la variable "expresión de género" (gender 
conforming o non-conforming) como "predictora" de experiencias de violencia, angustia, etc. Si identidad, género y sexualidad van de la mano a veces sí, a veces no, rara vez se presta atención al tema de la apariencia más o menos masculina de los varones y más o menos femenina de las mujeres (como quiera que masculina y femenina se definan) en tanto fuente de problemas para quienes no se conforman a lo esperado. La expresión de género (modales, tono de voz, vestimenta, hexis corporal, etc.) es crucial para entender padecimientos presentes en niños/as, adolescentes y jóvenes LGBT, padecimientos que potencialmente dejan huellas luego en la vida adulta. ¿Cómo se "gestiona" una experiencia de no-conformidad de género en un marco de desigualdad y privación socio-económica? Esto es particularmente crucial en ámbitos homo-sociales, como las pandillas barriales y otras - tema que nuevamente "forma sistema" ya que estos ámbitos son tanto capital social para quienes cuentan con pocos o nulos otros recursos, como espacios de potenciación de conductas de riesgo (conductas de riesgo que pueden ser perfectamente racionales, como las de vender drogas o sexo y así contribuir a las finanzas del hogar, cuando el acceso al mercado de trabajo y otras actividades socialmente aceptadas está vedado circunstancial o sistemáticamente).

Los estudios sobre temas puntuales (uso de drogas, suicido, auto imagen corporal, situación de calle, ejercicio de la prostitución, entre varios) ilustran no sólo de cada uno de esos temas, sino que dan pistas para pensar esos procesos más amplios que van de la desigualdad a la salud (pasando por la orientación sexual) y viceversa. De todos modos, la revisión vuelve a focalizar en los individuos, en el sentido de que los factores sociales e institucionales se traducen en "factores de estrés" que pueden promover "conductas de riesgo para la salud". Más allá de las correlaciones positivas entre algunos factores y algunas conductas, la laguna que inunda estos análisis es insistir en el locus individual de los impactos y las respuestas. Quizá por el enfoque adoptado (estudios epidemiológicos y encuestas sociales) o por el contexto (norteamericano) de los trabajos, las vinculaciones entre estructuras de desigualdad y outcomes de salud no son to- madas como centrales. Las mediaciones entre las condiciones estructurales, acciones individuales y resultados en términos de daño o prevención, desaparecen.

En esa línea, los autores proponen intervenciones que apuntan a los servicios de salud y discuten por ejemplo la conveniencia o no de proveer servicios específicos para adolescentes LGB, poniendo el eje en el sector salud y en las cualidades más o menos amigables de las y los profesionales. Así, proponen una serie de recomendaciones (respetar la confidencialidad, no presumir la heterosexualidad en los formularios y en el lenguaje con el cual dirigirse a las y los jóvenes usuarios de los servicios de salud, entre otros; Coker et al., 2010, p. 471), que solo resulta útil y apropiada en la medida en que está dirigida a profesionales individuales que atienden a jóvenes LGB, por lo que no puede sino focalizarse en individuos que tratan con individuos. En relación con las variables estructurales, aquí no hay dificultades que sean exclusivas de la sub-población LGB (o LGB pobres o marginales), sino que tienen que ver con los ámbitos especializados y limitados de intervención que muchas veces, incluso, frustran a los propios profesionales y servicios. Lo que sí podemos subrayar, una vez más, es cómo dar cuenta aun en esos ámbitos especializados y limitados de los modos en que las variables estructurales (las desigualdades socio-económicas, los recursos materiales y simbólicos desigualmente distribuidos de manera persistente) intervienen en la producción de padecimientos y en las capacidades diferenciales de dar respuesta a ellos.

Hay otro trabajo que reúne resultados y recomendaciones para poblaciones como los jóvenes LGBT. El trabajo HIV Interventions for Mostat-Risk Young People (Fondo de Población de Naciones Unidas [UNFPA], World Health Organization [WHO], UNAIDS, \& Inter-Agency Task Team on HIV and Young People, 2008) fue elaborado por la Inter-Agency Task Team on HIV and Young People, establecida en 2001 para fortalecer la eficacia de la respuesta global al sida en el contexto de las generaciones jóvenes, en el marco de las Naciones Unidas. El trabajo, corto (es un Guidance brief), es muy interesante y refleja una visión que tiende a la integralidad de 
las respuestas, algo que es más declamado que explicado en muchos documentos internacionales. En lo que sigue voy a sintetizar algunos de sus puntos y señalar lo que me parecen fortalezas y también alguna debilidad ligada al modo de pensar y encarar la cuestión de las vulnerabilidades y respuestas al VIH en este segmento de población.

El Brief considera central intervenir en y con las sub-poblaciones jóvenes con mayor probabilidad de infectarse, es decir las sub-poblaciones jóvenes de las sub-poblaciones objeto de estudios epidemiológicos e intervenciones a estas alturas ya bastante abordadas: "varones y mujeres que se inyectan drogas y comparten equipo no esterilizado"; "varones que tienen sexo anal sin protección con otros varones"; "mujeres y varones que están involucrados en trabajo sexual, incluyendo quienes son objeto de tráfico con fines de explotación sexual y tiene sexo comercial (a menudo explotativo) sin protección"; y "varones que tienen sexo no protegido con trabajadoras/ es sexuales".

El fragmento que acabo de citar muestra al mismo tiempo la fortaleza y la debilidad de esta propuesta: la fortaleza es apuntar a las prácticas concretas y a los sujetos "tal cual son", de modo tal de intervenir allí donde el VIH infecta y produce daños; la debilidad es poner nuevamente en el centro del análisis y la intervención a individuos y prácticas (o peor aún, conductas- behaviours) desagregando ficticiamente prácticas de sujetos y sujetos de sus relaciones sociales, colectivos, comunidades y trayectorias. Y no es sólo un problema de identidades que permitan a los sujetos reconocerse con mayor o menor transparencia y felicidad, con mayor o menor violencia. Sino que se trata de un recorte parcial de realidades que impiden comprender los fenómenos estructurales e históricos, explicar los patrones generales. Y las situaciones específicas, y pensar intervenciones eficaces.

El Brief, que tiene el mérito de poner el foco en la vulnerabilidad juvenil específicamente "HSH", al mismo tiempo insiste en la desagregación analítica: "Es la conducta [behaviour] la que pone a la persona joven en riesgo de VIH. Diferentes sub-grupos de gente joven pueden te- ner conductas de riesgo para el VIH y varían de país en país" (UNFPA et al., 2008, p. 2). Aun contextualizado, el locus de la intervención es la conducta de riesgo y no la estructura de desigualdades que la produce.

El Brief recuerda algunos "settings" como los centros de detención para jóvenes, en los que tienen lugar encuentros sexuales sin protección y uso inyectable de drogas. El texto habla de anal sex. Más allá del tipo de relación sexual, el énfasis está puesto en la conducta que transmite el VIH y no en settings que de por sí producen enfermedades, padecimientos y violencia.

Más allá de este énfasis en individuos y prácticas individuales (aun cuando todas esas prácticas son relacionales: se tiene sexo con otro/s, se comparte drogas con otro/s), el Brief señala correctamente que las vulnerabilidades juveniles, en este caso al VIH/sida, son estructuralmente producidas y reproducidas, como sintetizamos en los párrafos siguientes.

Un elemento es socio-político (son mis términos, no los del Brief): muchas de las prácticas que traen riesgos y daños para la salud de las y los jóvenes son, según los países y las edades, estigmatizadas, ilegales, clandestinas y castigadas. Aquí vamos apenas mencionar el tema del uso de drogas y su persecución, fenómeno cuyas consecuencias para los derechos y la salud de la juventud merece un capítulo aparte pero central cuando se trata de desigualdades socio-económicas y juventud. Sí vamos a insistir en que tanto las prácticas y los vínculos homosexuales, como las disidencias de género (identidad de género, expresión de género), en casi todos los países del mundo están penalizadas para menores de edad, aun entre menores, y en varios también lo está para los jóvenes mayores de edad; y en casi todos los países está estigmatizada y sancionada socialmente. Todo ello promueve directa o indirectamente riesgos y daños para la salud. El tema de la ilegalidad y de las "zonas grises" (es decir, zonas de semi-legalidad, de legalidades o prohibiciones ambiguas o ambivalentes, o de regulación oficiosa pero no oficial; Pecheny, 2010), no sólo impide dar respuestas mejores y más eficaces por ejemplo en salud sexual y reproductiva, sino que produce una "lógica de la sospecha" 
(Epele, 2010) que impide la comunicación entre jóvenes e instituciones (y el Estado) y produce daños evitables.

El Brief (UNFPA et al., 2008) termina con una serie de recomendaciones en las líneas conocidas y válidas. Aquí lo estructural aparece como barrera a la intervención, no como productor de la vulnerabilidad y el daño.

\section{Hacia un Balance de las Lagunas, Puntos de Indagación y Mis Propias Elucubraciones}

El estigma y clandestinidad sexuales sumados a la falta de espacios y recursos propios dan como resultado una situación específica, pero identificable, de vulnerabilidad en materia de salud. Vulnerabilidad que es socio-política: sistemática y universal, pero socio-política, no natural. Jóvenes LGBT de clases medias y altas comparten estos procesos de vulnerabilización ligados a su orientación sexual e identidad de género no esperables según patrones de heteronormatividad. Pero en jóvenes LGBT marginales, pobres y en contextos de desigualdad económica, estos procesos producen casi inexorablemente condiciones de morbilidad y mortalidad precoces. Siguen haciendo falta investigaciones empíricas que den cuenta de estas producciones diferenciales de vulnerabilidad generacional según clase, género, orientación sexual, identidad y expresión de género, y los otros clivajes etnicidad, raza, etc.

Podemos preguntarnos cómo operan los contextos de desigualdad económica, pobreza y marginalidad, de manera diferencial para jóvenes mujeres heterosexuales, jóvenes varones heterosexuales, y LGBT (cada categoría individualmente considerada), en términos de permanencia en la vivienda y familia de crianza, constitución de familias ampliadas, acceso al mercado de trabajo, proyectos de mater-paternidad, participación en la provisión de ingresos al hogar, etc.; y en qué medida y cómo estos procesos tienen impacto a la salud de jóvenes LGBT.

La pregunta a responder empíricamente es cómo entender el papel productor de riesgos y daños que tienen las desigualdades, y cómo son los procesos concretos en que se dan estos fenó- menos. La intersección de juventud y orientaciones sexuales e identidades LGBT especifica esos "cómo", en un "combo": desigualdad estructural e histórica en cuyo marco las y los jóvenes que carecen de recursos asumen comportamientos que pueden parecer (y ser a veces) irracionales o nocivos pero que tienen sus lógicas en contextos de pocas o nulas opciones de ingreso al mercado de trabajo, la protección social (usualmente estructurada ya sea al trabajo formal, ya sea en torno a la noción heteronormativa de familia y la madre a cargo de hijos/as) o las instituciones educativas y de salud, y de relación ciudadana con un Estado ausente o semi-ausente, o presente solo en calidad de represor y articulador del clientelismo, la corrupción o el crimen.

En algunos países de manera endémica, en otros de manera cíclica más o menos previsible o inesperada, el Estado "desaparece" y deja instituidas zonas de exclusión, de marginalidad, de no-Estado y por ende de lazos sociales más endebles y violentos. La temporalidad aquí desaparece en un hoy permanente, en un presente sin pasado y sin futuro en el que solo vale sobrevivir el día. ¿Cómo pensar aquí en instalar comportamientos saludables, proyectos a mediano y largo plazo, reconocer a los individuos y grupos como sujetos de sus propias vidas? En los contextos de violencia, rotura de redes sociales y desamparo estatal que constituyen verdaderos "estados de naturaleza hobbesianos", las y los jóvenes desarrollan sus estrategias (o conductas reactivas) de supervivencia y es lícito hipotetizar que las y los jóvenes LGBT tendrán, como todos, sus estrategias y conductas específicas. Para muchas/os, el recurso al sexo compensado es una estrategia no solo tolerada, sino alentada o forzada por las propias familias en situación de penuria económica. El uso y la venta de drogas, algunas muy nocivas, el arriesgar la vida en pequeños o grandes delitos cuando la vida ya está en riesgo de muerte prematura por el solo hecho de transitar barrios y zonas "de nadie", caracterizan a vastos sectores de jóvenes y aun de niños.

Ser un/a joven LGBT agrega dimensiones suplementarias que, más que yuxtaponerse, complejizan y matizan estos procesos de vulnerabilidad social de jóvenes: en algunos casos 
para empeorar y en otros, paradójicamente, para abrir válvulas de escape. La salida relativamente más temprana de la vivienda familiar, la exogamia de clase ligada a la endogamia de los vínculos socio-sexuales, la resiliencia practicada en el seno de los propios núcleos de socialización potencial o realmente hostiles ante un niño/a o adolescente que escapa a la heterosexualidad o identidad de género esperada, entre otras posibles experiencias, pueden alentar la ruptura con ciclos de violencia, pauperización y exclusión, que por ejemplo los varones heterosexuales socializados en ambientes de "no futuro".

En síntesis, casi todos los trabajos encontrados parten de, y reproducen, una matriz individualista en las concepciones de la génesis de los problemas, de la investigación y de las respuestas recomendadas. Si las políticas se tienen que basar en evidencia, si monitorear y evaluar intervenciones implican evidencia, la noción misma de qué entendemos por evidencia científica tiene que ser puesta en cuestión.

La presunción de heterosexualidad como barrera de exclusión institucional y de socialización es un tema para desarrollar en relación con los procesos sociales de desigualdad y en relación con la salud (Altman et al., 2012). ¿Es esta presunción un factor que repercute en términos de movilidad social? ¿Y en qué dirección?

Algunos esfuerzos, aunque no tan sistemáticos, se han hecho en el seno de "la comunidad de respuestas al VIH", como el trabajo aquí reproducido del equipo inter-agencial. Pero por ejemplo allí se habla de jóvenes HSH (más allá de la polémica por la denominación) y no de las lesbianas, como si la heteronormatividad y desigualdad sexo-genérica fuera sólo una cuestión de varones (y trans) y por el VIH/las ITS solamente. Pero si no es el VIH/sida un (gran) problema para las jóvenes lesbianas, ¿por qué esperar a que surja un "gran" problema específico de las jóvenes lesbianas y bisexuales para atacar a las condiciones estructurales generativas de riesgos y daños?

Dicho todo lo que hemos dicho, igual cabe preguntarse si la "no separación" de LGBT del resto de los jóvenes no puede ser algo bueno en el mediano y largo plazo. Es decir, combatir la producción estructural de vulnerabilidad de jó- venes LGBT no implica en realidad combatir la producción estructural de vulnerabilidad en todos los jóvenes y alentar la diversidad y respeto en todos los órdenes de la vida de dichos jóvenes.

Por último, nos cuesta ver las dimensiones sexuadas y económicas de las relaciones sociales no tan explícitamente sexuadas ni económicas. Por ejemplo en qué medida existe una totalidad de relaciones sociales con dimensiones de mercado, género (incluyendo identidad y expresión), sexualidad (incluyendo prácticas y orientaciones), clase, etc. que "producen" de manera dialéctica (vale la pena volver a Adorno en su polémica con Popper) una totalidad cuyos momentos particulares solo se comprenden y explican por esa totalidad.

En síntesis, lagunas hay. No creo que ellas se expliquen por falta de fondos y prioridades, solamente. Sino por dos problemas "extremos" (en un continuo en que las metodologías y técnicas de investigación han avanzado mucho): un extremo, la epistemología; el otro extremo, las técnicas de intervención. Las epistemologías positivistas, que tienden a analizar (es decir, desagregar la realidad), terminan en muchos casos brindando pinturas muy parciales de los fenómenos que pretenden describir, explicar y comprender. Una vuelta al espíritu dialéctico de la "totalidad concreta" (cuyos momentos "universales abstractos" y "particulares concretos" son momentos de un programa sintético de conocimiento) no vendría mal. $\mathrm{Y}$ en cuanto a técnicas de intervención: ¿cómo pensar y poner en práctica técnicas eficaces, en el sentido más amplio de esta palabra, que no sean "ingeniería social fragmentaria" en el sentido popperiano?

\section{Referencias}

Altman, D., Aggleton, P., Williams, M., Kong, T., Reddy, V., Harrad, D., ...Parker, R. (2012). Men who have sex with men: Stigma and discrimination. Lancet, 380, 439-445.

Auerbach, J. D., Parkhurst, J. O., \& Cáceres, C. F. (2011). Addressing social drivers of HIV/AIDS for the long-term response: Conceptual and methodological considerations. Global Public Health, 6(Suppl. 3), S293-S309. 
Ayres, J. R. (2002). Conceptos y prácticas en salud pública: algunas reflexiones. Revista de la Facultad Nacional de Salud Pública, 20(2), 67-82.

Ayres, J. R., França, I., Jr., Junqueira Calazans, G., \& Saletti, H. C., Filho. (2008). El concepto de vulnerabilidad y las prácticas de salud: nuevas perspectivas y desafíos. In N. Almeida Filho \& R. Fernandez Silva, Promoción de la salud: conceptos, reflexiones, tendencias (pp. 135-161). Buenos Aires, Argentina: Lugar Editorial.

Ayres, J. R., Paiva, V., \& França, I., Jr. (2012). Conceitos e praticas de prevenção: Da historia natural da doença ao quadro da vulnerabilidade e direitos humanos. In V. Paiva, J. R. Ayres, \& C. M. Buchalla (Coords.), Vulnerabilidade e direitos humanos. Prevenção e promoção da saúde: Vol. I. Da doença a cidadania (pp. 71-94). San Pablo, SP: Jurúa.

Beyrer, C., Baral, S. D, van Griensven, F., Goodreau, S., Chariyalertsak, S., Wirtz, A., ...Brookmeyer, R. (2012). Global epidemiology of HIV infection in men who have sex with men. Lancet, 380(9839), 367-377.

Coker, T. R., Austin, S. B., \& Schuster, M. A. (2010). The health and health care of lesbian, gay and bisexual adolescents. Annual Review of Public Health, 31, 457-477.

Epele, M. (2010). Sujetar por la herida. Buenos Aires, Argentina: Paidós.

Facchini, R., \& Barbosa, M. R. (2006). Saúde das mulheres lésbicas. Promoção da equidade $e$ da integralidade. Recuperado em http://www. redesaude.org.br/Homepage/Dossi\%EAs/ Dossi\%EA\%20Sa\%FAde\%20das\%20Mulheres $\% 20 \mathrm{~L} \%$ E9sbicas.pdf

Fondo de Población de Naciones Unidas, World Health Organization, Joint United Nations Program on HIV/AIDS, \& Inter-Agency Task Team on HIV and Young People. (2008). HIV interventions for most-at-risk young people. Geneva, Switzerland: Inter-Agency Task Team on HIV and Young People. Retrieved from http://www. unfpa.org/hiv/iatt/docs/mostatrisk.pdf

Habermas, J. (1988). La lógica de las ciencias sociales. Madrid, España: Tecnos.

Illouz, E. (2009). El consumo de la utopía romántica. El amor y las contradicciones culturales del capitalismo. Buenos Aires, Argentina: Katz.
Joint United Nations Program on HIV/AIDS. (2011). Synthesis of strategic information on HIV and young people. Geneva, Switzerland: Author.

Programa Conjunto de las Naciones Unidas sobre el VIH/Sida. (2006). Derechos humanos, salud y VIH. Guía de acciones estratégicas para prevenir y combatir la discriminación por orientación sexual e identidad de género. Recuperado em http://www.onusida.org.co/Derechos\%20humanos $\% 20$ salud $\% 20 \mathrm{y} \% 20$ VIH.pdf

Padilla, M., Vázquez del Aguila, E., \& Parker, R. (2007). Globalization, structural violence, and LGBT health: A cross-cultural perspective. In I. H. Meyer \& M. E. Northridge (Eds.), The health of sexual minorities (pp. 209-241). New York: Springer.

Paiva, V., Ayres, J. R., \& Buchalla, C. M. (Orgs.). (2012). Vulnerabilidade e Direitos Humanos. Prevenção e promoção à saúde: Da doença à cidadania. San Pablo, SP: Juruá.

Parker, R., \& Aggleton, P. (2003). HIV and AIDSrelated stigma and discrimination: A conceptual framework and implications for action. Social Science and Medicine, 57, 13-24.

Pecheny, M. (2002). Identidades discretas. In L. Arfuch (Org.), Identidades, sujetos y subjetividades (pp. 125-147). Buenos Aires, Argentina: Prometeo.

Pecheny, M. (2010). Emergency contraception in Argentina: Grey areas of common sense and public policy. Global Public Health Journal, 5(3), $1-14$.

Pecheny, M., \& de la Dehesa, R. (2012). Sexualidades, Política e Estado na América Latina: Elementos críticos a partir de um debate Sul-Sul. Polis e Psique (Porto Alegre), 1(3), 26-64.

Petchesky, R. (2007). Sexual rights policies across countries and cultures: Conceptual frameworks and minefields. In R. Parker, R. Petchesky, \& R. Sember (Eds.), Sex politics: Reports from the front line. Nueva York: Sexuality Policy Watch.

Remafedi, G. (2007). Health disparities for homosexual youth: The children left behind. In R. Wolitsky, R. O. Valdiserri, \& R. Stall (Eds.), Unequal opportunity: Health disparities affecting gay and bisexual men in the United States (pp. 275-301). New York: Oxford University Press. 
Stall, R., Friedman, M., \& Catania, J. A. (2007). Interacting epidemics and gay men's health: A Theory of Syndemic Production among urban gay men. In R. Wolitsky, R. O. Valdiserri, \& Stall, R. (Eds.), Unequal opportunity: Health disparities affecting gay and bisexual men in the United States (pp. 251-274). New York: Oxford University Press.
Wong, C. F., Weiss, G., Ayala, G., \& Kipke, M. D. (2010). Harassment, discrimination, violence, and illicit drug use among young men who have sex with men. AIDS Education and Prevention, 22(4), 286-298. 